\title{
Spectroscopic rotation velocities of $L$ dwarfs from VLT/UVES and their comparison with periods from photometric monitoring ${ }^{\star}$
}

\author{
C. A. L. Bailer-Jones ${ }^{1,2, \star \star, \star \star \star}$ \\ ${ }^{1}$ Max-Planck-Institut für Astronomie, Königstuhl 17, 69117 Heidelberg, Germany \\ ${ }^{2}$ Carnegie Mellon University, Department of Physics, 5000 Forbes Ave., Pittsburgh, PA 15213, USA
}

Received 3 January 2004 / Accepted 23 February 2004

\begin{abstract}
The variability and rotation of ultra cool dwarfs (UCDs) provide important information on the atmospheres and evolution of these very low mass stars and brown dwarfs. As part of an ongoing program to investigate this, the projected rotation velocities, $v \sin i$, derived from high resolution VLT/UVES spectroscopy via cross correlation are presented for 16 field UCDs (M9V-L7.5V). This doubles the number of $\mathrm{L}$ dwarfs for which $v \sin i$ has been measured. All targets are found to have $v \sin i$ between 10 and $40 \mathrm{~km} \mathrm{~s}^{-1}$ confirming that $\mathrm{L}$ dwarfs are rapid rotators. Radial velocities have also been measured to a precision of $1-2 \mathrm{~km} \mathrm{~s}^{-1}$. From the random distribution of the rotation axes, $i$, and theoretically predicted radii, one-sided confidence intervals are placed on the rotation periods of individual objects. These are compared with published period data obtained from photometric monitoring programs. From this, the period of $31 \mathrm{~h}$ for the L 0.5 dwarf $2 \mathrm{M} 0746+2000$ published by Gelino et al. (2002) may be ruled out as the rotation period. The period of $11.2 \pm 0.8 \mathrm{~h}$ for the L1.5 dwarf $2 \mathrm{M} 1145+2317$ obtained by Bailer-Jones \& Mundt (2001) is consistent with the present $v \sin i$ results so is plausibly the true rotation period. The inclination of the rotation axis is constrained to be $i=62^{\circ}-90^{\circ}$ with an expectation value of $76^{\circ}$. Alternatively the data set a lower limit on the radius of $0.1 R_{\odot}$, which is within the range of radii predicted by models for brown dwarfs older than 0.5 Gyr. Similarly, the period of $2.7 \pm 0.1 \mathrm{~h}$ detected by the same authors for $2 \mathrm{M} 1334+1940$ is also confirmed as the likely rotation period; the inclination is $i=27^{\circ}-44^{\circ}\left(\langle i\rangle=34^{\circ}\right)$. Where no variability or period was detected by the monitoring programs the likely reason is low contrast modulating surface features. However, in three cases variability but no period was detected, even though the likely rotation period range inferred from $v \sin i$ lies within the timescale to which the monitoring was sensitive. This reinforces the "masking hypothesis" of Bailer-Jones \& Mundt (2001), the idea that the evolution of photospheric features on timescales shorter than the rotation period obscure the regular modulation of the light curve. As has been previously discussed, a likely candidate for such features is inhomogeneous dust clouds.
\end{abstract}

Key words. techniques: spectroscopic - stars: low-mass, brown dwarfs - stars: variables: general - stars: rotation

\section{Introduction}

Understanding the nature of low mass stars and brown dwarfs requires a detailed characterization of their observable atmospheres. Specifically, the determination of luminosities, chemical compositions and ages ultimately depends on adequate knowledge of the radiative and convective transport mechanisms. While our understanding of these mechanisms is reasonable (if incomplete) for some types of stars, it is comparatively poor for ultra cool dwarfs (UCDs), late type M, L and T dwarfs. This is not least because at the low effective temperatures involved $(<3000 \mathrm{~K})$, solid dust particles form in significant numbers and varieties, and these have a major impact on the structure of the atmosphere and hence on the observable properties of the star (e.g. Allard et al. 2001; Burrows \& Sharp 1999). In

\footnotetext{
* Based on observations obtained at the VLT, European Southern Observatory, Chile, program 65.L-0199.

$\star \star$ Emmy Noether Fellow of the Deutsche Forschungsgemeinschaft. $\star \star \star$ e-mail: calj@mpia-hd.mpg.de
}

particular, global dust structures (“clouds") may play a significant role.

Significant information on UCDs can be obtained from their observable temporal changes, and several dedicated monitoring programs have now uncovered good evidence for optical and infrared variability (Bailer-Jones \& Mundt 1999, 2001; Terndrup et al. 1999; Tinney \& Tolley 1999; Nakajima et al. 2000; Martin et al. 2001; Bailer-Jones 2002; Burgasser et al. 2002; Clarke et al. 2002a, 2002b, 2003; Gelino 2002; Gelino et al. 2002; Bailer-Jones \& Lamm 2003; Enoch et al. 2003; Joergens et al. 2003; Koen 2003; Zapatero Osorio et al. 2003) and $\mathrm{H} \alpha$ variability (Hall 2002; Mochnacki et al. 2002; Liebert et al. 2003). Of the 80 or so UCDs monitored by these groups, about 30 show evidence for photometric variability. While this figure depends on what one takes as sufficient evidence for variability, at least half are convincingly variable on the scale of a few tens of millimagnitudes. There are indications that this photometric variability is not caused by a simple rotational modulation of a non-uniform photosphere. 
One candidate is an inhomogeneous dust layer evolving under the influence of convection on time scales shorter than the rotation period (see the introduction to Bailer-Jones 2002 for a discussion). Understanding this phenomenon is important, not only for what it tells us about atmospheric process under these interesting physical conditions, but also for the implications it has concerning conclusions drawn from single epoch observations of UCDs.

In this article I address the specific issue of how the photometric variability and period data relate to UCD rotation, as measured by line broadening from high resolution spectroscopy. This provides the projected rotation speed, $v \sin i$, so using simple statistical arguments and theoretical predictions for UCD radii, constraints can be placed on likely rotation periods. These (plus other published $v \sin i$ data) are compared with the variability data to confirm or refute possible rotation periods and investigate the "masking hypothesis".

Previously published work has established that UCDs are fast rotators: of the 17 hitherto observed L dwarfs, 16 have $v \sin i$ in the range $10-40 \mathrm{~km} \mathrm{~s}^{-1}$ and one (Kelu-1) has $60 \mathrm{~km} \mathrm{~s}^{-1}$ (Basri et al. 2000; Mohanty \& Basri 2003; Schweitzer et al. 2001). In the present work, $v \sin i$ values are determined for 16 UCDs (15 L dwarfs and $1 \mathrm{M}$ dwarf), 14 of which have no previously published measurement. Moreover, conservative ranges for $v \sin i$ are established which reflect the dominant uncertainties in measuring $v \sin i$ by cross correlation. For the majority of UCDs, the complete $v \sin i$ range lies between 10 and $30 \mathrm{~km} \mathrm{~s}^{-1}$, and there is no measurement below about $10 \mathrm{~km} \mathrm{~s}^{-1}$, thus confirming the rapid rotation of UCDs. For a discussion of UCD rotation and its relation to chromospheric activity and possible dynamo mechanisms, the reader is referred to Mohanty \& Basri (2003).

\section{Data acquisition and reduction}

A target list of UCDs ranging in spectral type from M9 to L8 was assembled from the published UCDs available at the time of the observations (April 2000). They were selected preferentially for brightness and for good observability from the VLT and to cover a range of spectral types. They were not selected based on any known $v \sin i$ values (none were available at the time), although some L dwarfs monitored by Bailer-Jones \& Mundt (1999, 2001) were specifically included.

\subsection{Instrumentation and observations}

High resolution spectroscopy was obtained with the UVES echelle spectrograph mounted on the UT2 $8.2 \mathrm{~m}$ VLT telescope at Cerro Paranal, Chile, on 26-28 April 2000. The red arm of this instrument was used with the CD4-prot cross dispersion grating and a slit width of $1^{\prime \prime}$. This provides the wavelength range 6440-10250 $\AA$ (orders 94-60) at a resolution of 38700 , yielding a $F W H M$ of $7.8 \mathrm{~km} \mathrm{~s}^{-1}$. The detector is a mosaic of two CCDs with pixels binned to provide a sampling of $2.4 \mathrm{~km} \mathrm{~s}^{-1} /$ pix in the dispersion direction and $0.35^{\prime \prime} /$ pix in the spatial direction. The slit length was $10^{\prime \prime}$ and was oriented vertically (i.e. parallel to the direction of maximum atmospheric dispersion).
Over the course of two and a half nights, spectra were obtained of the 16 UCDs listed in Table 2. Exposure times ranged from $1 \times 15 \mathrm{~min}$ for the brightest targets to $3 \times 50 \mathrm{~min}$ for the faintest targets. The seeing was typically $0.8^{\prime \prime}\left(0.5^{\prime \prime}-1.0^{\prime \prime}\right)$ at airmasses of up to 2.0. Spectra were also obtained several times with the same instrument settings for $v \sin i$ and $v_{\text {rad }}$ standards, namely the bright $\mathrm{M}$ dwarfs G1402, G1406 and G1876. The spectrograph is mounted on the Nasmyth platform so undergoes limited movement during the course of the observations. The data reduction procedure verified the stability of the instrument. While wavelength calibration spectra and flat fields were taken at regular intervals each night, it was found that a single set of calibration frames for each night was sufficient.

\subsection{Data reduction and spectral extraction}

The objective of the data reduction is to produce wavelength calibrated one-dimensional spectra for each echelle order, free of relevant instrumental and telluric signatures as preparation for cross correlation. The basic data reduction was carried out using the IRAF ${ }^{1}$ package, and consists of the removal of scattered light, flat fielding, spectral extraction and wavelength calibration.

The two-dimensional data format (i.e. cross dispersed spectra) will be referred to as an "image". Scattered light in the spectrograph contributes a two-dimensional additive pattern to the dispersed light and generally needs to be removed from both science frames and flats. This was done by tracing the orders and defining the inter-order regions. A two-dimensional fit is made to these regions and this fit subtracted from all images. As dark frames, biases and overscan regions showed no features, a further explicit subtraction of these was not necessary.

Flat fielding is performed to remove small scale detector variations and fringing ${ }^{2}$. A sequence of individual flat field images is combined. This master flat was then rectified using the APFLATTEN task, which makes fits to each order to remove the pseudo-continuum and grating blaze function. The result is a two-dimensional flat field preserving only high frequency variations which are then removed from the science frames by division. The global variations (pseudo-continuum, blaze function etc.) are removed later immediately prior to cross correlation.

The spectra are extracted and the sky subtracted using the APSUM package. A one-dimensional optimal extraction was performed with pixel cleaning based on expected noise statistics. Because many of the $\mathrm{L}$ dwarfs are faint, extraction

1 The Image Reduction and Analysis Facility, provided by the National Optical Astronomy Observatories (NOAO).

${ }^{2}$ In broad band direct imaging, fringing (monochromatic interference in the $\mathrm{CCD}$ ) is generated by night sky line emission sources. As discussed in Bailer-Jones \& Mundt (2001) this produces an additive pattern over the whole CCD which must be subtracted to perform correct photometry (and not divided, as is often thought, because it does not modulate the star light). With high resolution spectroscopy the situation is different. Here the grating itself creates an independent narrow band source at every point along the dispersion direction which in turn generates fringing at each point in the CCD. All incident sources therefore create the same fringe pattern at a given wavelength, so the fringes must be divided out using a flat field. 
apertures are not traced: they are transferred from the order definition image and simply recentered and resized to accommodate both the position of the sources in the slit and the seeing.

A wavelength calibration was established using the internal ThAr lamp. Lines were iteratively identified and a fit produced using the ECREIDENTIFY package. The fit was a twodimensional Chebyshev polynomial based on the spectral order numbers (using a third order polynomial along the dispersion axis and second order one perpendicular to it). The rms of the fit was 0.01 pixels, or $25 \mathrm{~m} / \mathrm{s}$. This was applied to the science spectra using the DISPCOR package. Changes in temperature or pressure or seismic disturbances can alter the zero point of this calibration during the course of a night. The calibration was therefore checked in all science images against the positions of the $\mathrm{OH}$ emission lines from the Earth's atmosphere. These demonstrated that the zero point shift of the instrumental calibration was below the rms of the fit. Of course, additional zero point shifts occur in the applied wavelength scale if a star is not perfectly centered in slit, and could be as large as the projected slit size. In practice, though, autoguiding keeps the star centered in the slit to within about a tenth of the FWHM, giving an upper limit on the systematic zero point shift (and hence systematic radial velocity error) of about $1 \mathrm{~km} \mathrm{~s}^{-1}$. This does not effect the rotational velocities.

Candidate wavelength regions for the cross correlation were selected on the grounds of being relatively free of telluric absorption, as identified via inspection of stellar spectra with few intrinsic features (a rapidly rotating B9V, a B5V and an A0III) acquired for this purpose. As a result, just those orders (or parts of orders) listed in Table 1 are retained for all targets and templates. Regions containing strong telluric emission lines (which, on account of noise, are only poorly removed by sky subtraction) were likewise removed. While performing the cross correlations on individual objects, all orders were visually inspected and any obvious emission lines, cosmic rays or bad pixels were masked. Not all orders in Table 1 were used to form the final values of $v \sin i$ or $v_{\text {rad }}$ : orders were selected for each star separately based on the quality of the cross correlation function (see below). Example spectra for three objects are shown in Fig. 1. The signal-to-noise ratio (SNR) varies for different orders and different targets, but in order 70 it is $8-25$ per extracted pixel $(0.05 \AA$; Cf. $F W H M$ of a resolution element is $0.23 \AA$ in order 70). Orders with $S N R$ lower than a few were disregarded; for this reason the bluest orders were often not used.

\section{Radial velocities}

Radial velocities are determined via cross correlation of the UCD spectra with the spectrum of an observed star of known radial velocity. This was done in two stages. The initial template used was G1406, an M4V star with $v_{\text {rad }}$ reported as $19.48 \pm$ $0.03 \mathrm{~km} \mathrm{~s}^{-1}$ by Nidever et al. (2002) and $19.18 \pm 0.11 \mathrm{~km} \mathrm{~s}^{-1}$ by White \& Basri (2003). I adopt the former. The cross correlation was performed using the IRAF package FXCOR, with the centre of the cross correlation peak calculated using the "center1d" option. The pseudo-continuum of each order is rectified (fit and divided out) using a fifth order cubic spline.
Table 1. Candidate orders or partial orders retained for the cross correlation. In individual cases, some orders produce poor correlation functions in either the cross correlation of the template against the target or, for $v \sin i$, the template against the spun up template (calibration), in which case the order is not used. Orders 90-94 were not used with the $\mathrm{L}$ dwarf rotation template, and only orders $60-72$ were use in determining $v_{\text {rad }}$, in both cases for $S N R$ reasons.

\begin{tabular}{lrr}
\hline \hline Order & $\lambda_{\text {min. } / \AA}$ & $\lambda_{\text {max. } / \AA}$ \\
\hline 60 & 10095.0 & 10250.0 \\
61 & 9931.0 & 10080.0 \\
62 & 9850.0 & 9917.0 \\
69 & 8786.0 & 8903.0 \\
70 & 8661.0 & 8775.0 \\
71 & 8540.0 & 8651.0 \\
72 & 8419.0 & 8530.0 \\
75 & 8087.0 & 8130.0 \\
76 & 7979.5 & 8078.0 \\
77 & 7877.0 & 7973.0 \\
78 & 7777.0 & 7869.0 \\
79 & 7699.0 & 7770.0 \\
81 & 7490.0 & 7577.0 \\
82 & 7399.0 & 7484.0 \\
85 & 7139.0 & 7165.0 \\
86 & 7054.0 & 7133.0 \\
89 & 6820.0 & 6866.0 \\
90 & 6745.0 & 6815.0 \\
91 & 6670.0 & 6739.0 \\
92 & 6598.0 & 6665.0 \\
94 & 6460.0 & 6523.0 \\
\hline
\end{tabular}

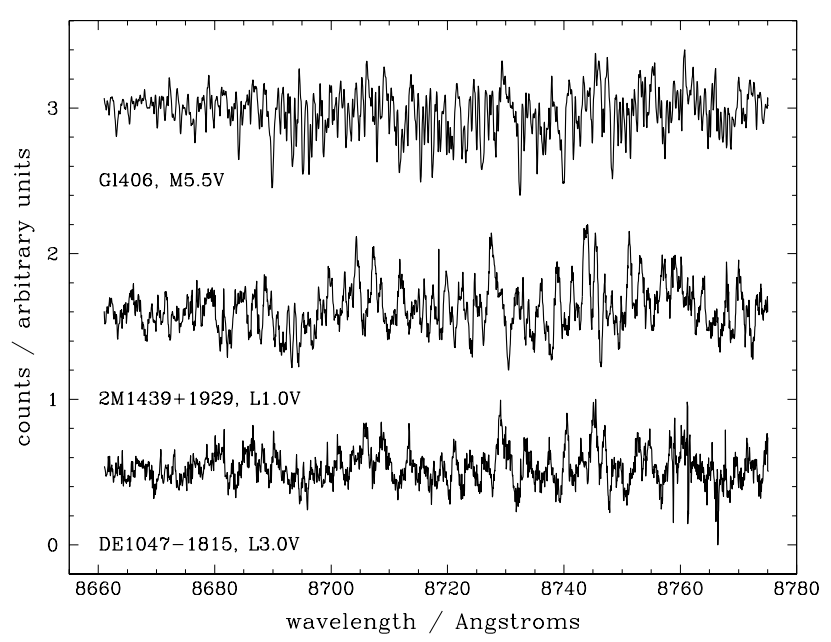

Fig. 1. Spectra (order 70) of an $\mathrm{M}$ dwarf $v \sin i$ and $v_{\text {rad }}$ standard (top), an L1.0 dwarf (also the L dwarf "standard", middle), and an L3.0 dwarf (bottom). The spectra have been continuum subtracted, scaled to a common vertical range and offset on the vertical axis. The exposure times for these single spectra were $3 \mathrm{~min}, 15 \mathrm{~min}$ and $40 \mathrm{~min}$ respectively, which achieve mean $S N R$ per extracted pixel around the centre of the order of 150,15 and 11 respectively. 
Table 2. $v \sin i$ values for ultra cool dwarfs observed in this survey. The first column lists the full name of each target: an abbreviated version is used elsewhere in this paper, where $2 \mathrm{M}=2 \mathrm{MASS}, \mathrm{DE}=\mathrm{DENIS}$ and $\mathrm{SD}=\mathrm{SDSS}$. The second and third columns list the spectral types and I magnitudes taken from the literature. (I magnitudes given to only one decimal place have been estimated.) Columns 4-7 and 8-11 list the values of $v \sin i$ deduced via cross correlation with the M dwarf templates and L dwarf template (2M1439+1929) respectively. "mean" (i.e. $\overline{v \sin i}$ ) is a weighted mean formed by combining measurements from individual spectrograph orders and/or exposures: $N$ orders are retained in the final calculation. "min." and "max." are the minimum and maximum values of $v \sin i$ similarly averaged over all orders, and so encompass generous estimates of the uncertainty in fitting the peak of the cross correlation function. The final column gives the radial velocities relative to the solar system barycentre. In addition to the random errors given for these, there may be an additional systematic error from the zero point uncertainty (due to possible slit decentering) of no more than $1 \mathrm{~km} \mathrm{~s}^{-1}$. A colon, "“:”, indicates an uncertain value.

\begin{tabular}{|c|c|c|c|c|c|c|c|c|c|c|c|c|}
\hline \multirow[t]{3}{*}{ Name } & & \multirow[t]{3}{*}{ SpT } & \multirow[t]{3}{*}{$I$} & \multicolumn{8}{|c|}{$\overline{v e \sin i / \mathrm{km} \mathrm{s}^{-1}}$} & \multirow[t]{3}{*}{$\overline{v_{\mathrm{rad}} / \mathrm{km} \mathrm{s}^{-1}}$} \\
\hline & & & & \multicolumn{4}{|c|}{ M dwarf template } & \multicolumn{4}{|c|}{ 2M1439+1929 template } & \\
\hline & & & & mean & $\min$. & $\max$. & $N$ & mean & $\min$. & $\max$. & $N$ & \\
\hline DENIS-P & J1431-1953 & M9.0 & 17.47 & 47.4 & 44.1 & 52.6 & 1 & 37.1 & 33.7 & 41.6 & 4 & $-11.4 \pm 2.8$ \\
\hline DENIS-P & $\mathrm{J} 1159+0057$ & L0.0 & 17.32 & 35.2 & 31.9 & 39.7 & 3 & 74.5 & 69.1 & 83.5 & 1 & $-3.0 \pm 1.5:$ \\
\hline 2MASSI & $\mathrm{J} 0746425+200032$ & L0.5 & 15.11 & 27.3 & 25.6 & 30.6 & 6 & 25.8 & 23.0 & 28.8 & 9 & $54.1 \pm 0.9$ \\
\hline 2MASSW & $\mathrm{J} 1412244+163312$ & L0.5 & 17.1 & 17.3 & 14.7 & 19.2 & 6 & 16.4 & 13.9 & 19.2 & 4 & $6.9 \pm 0.8$ \\
\hline 2MASSW & J1439284+192915 & L1.0 & 16.12 & 11.2 & 9.6 & 12.8 & 9 & - & - & - & - & $-26.3 \pm 0.5$ \\
\hline DENIS-P & $\mathrm{J} 1441-0945$ & $\mathrm{~L} 1.0$ & 17.32 & 17.4 & 14.8 & 20.6 & 4 & 15.9 & 13.3 & 19.3 & 6 & $-27.9 \pm 1.2$ \\
\hline 2MASSW & $\mathrm{J} 1145572+231730$ & L1.5 & 18.62 & 12.5 & 10.5 & 14.4 & 9 & 12.7 & 10.5 & 14.8 & 6 & $3.7 \pm 0.9$ \\
\hline 2MASSW & $\mathrm{J} 1334062+194034$ & L1.5 & 18.76 & 25.2 & 22.1 & 28.1 & 4 & 25.4 & 21.5 & 29.9 & 5 & $-4.3 \pm 2.1:$ \\
\hline 2MASSI & $\mathrm{J} 1029216+162652$ & $\mathrm{~L} 2.5$ & 17.9 & 28.3 & 23.6 & 32.5 & 7 & 28.0 & 22.8 & 33.1 & 8 & $-29.2 \pm 4.0$ \\
\hline DENIS-P & $\mathrm{J} 1047-1815$ & $\mathrm{~L} 2.5$ & 17.75 & 16.3 & 15.0 & 18.3 & 12 & 15.0 & 12.2 & 17.6 & 11 & $6.0 \pm 0.8$ \\
\hline 2MASSW & J0913032+184150 & L3.0 & 19.07 & 15.0 & 11.9 & 17.6 & 11 & 20.3 & 18.0 & 22.6 & 3 & $28.4 \pm 2.5:$ \\
\hline SDSSp & $\mathrm{J} 120358.19+001550.33$ & L3.0 & 18.88 & 27.6 & 24.1 & 32.3 & 7 & 31.7 & 25.6 & 36.8 & 8 & $-2.7 \pm 2.3$ \\
\hline 2MASSW & $\mathrm{J} 1615441+355900$ & L3.0 & 18.1 & 12.1 & 10.7 & 13.8 & 5 & 12.8 & 9.4 & 16.0 & 10 & $-20.2 \pm 0.9$ \\
\hline 2MASSW & J2224438-015852 & L4.5 & 18.02 & 25.4 & 22.2 & 27.9 & 3 & 24.7 & 20.9 & 29.2 & 6 & $-37.4 \pm 3.4$ \\
\hline 2MASSW & $\mathrm{J} 1507476-162738$ & L5.0 & 16.65 & 27.1 & 22.6 & 31.6 & 4 & 27.2 & 23.4 & 32.7 & 4 & $-39.3 \pm 1.5$ \\
\hline 2MASSI & J0825196+211552 & L7.5 & 19.22 & - & - & - & - & 16.9 & 11.3 & 21.4 & 6 & $20.5 \pm 2.0$ \\
\hline
\end{tabular}

The cross correlation function was sometime quite noisy, in particular for the bluer spectral orders and for late L spectral types, resulting in ambiguous peak centering or even identification. For this reason, the bright L1.0 dwarf 2M1439+1929 was adopted as a "secondary" radial velocity standard, and only the orders in the "red" CCD (orders 60, 61, 62, 69, 70, 71 and 72 in Table 1) are used. The radial velocity of $2 \mathrm{M} 1439+1929$ is determined to be $-26.3 \pm 0.50 \mathrm{~km} \mathrm{~s}^{-1}$ from cross correlation against G1406. (The bright L0.5 dwarf 2M0746+2000 could also have been used for this purpose, but was not on account of its binarity; Sect. 7). The radial velocities for all other UCDs are determined by cross correlation against 2M1439+1929 and are listed in Table 2: the value given is a weighted mean of the results in the different orders. The uncertainty is the standard deviation in this mean (including the uncertainty in the template velocity), apart from for a few cases in which the peak centering uncertainty was larger, in which case this is reported instead.

Three of the results are dubious (marked with a ":") because of discrepancies between orders and/or significant centering uncertainties due to highly rotationally broadened spectral lines. The latter is particularly applicable for DE1159+0057 and DE1431-1953. Two of the targets have $v_{\text {rad }}$ determinations in the literature. Basri et al. (2000) obtained $v_{\text {rad }}=29 \pm 1 \mathrm{~km} \mathrm{~s}^{-1}$ for $2 \mathrm{M} 1439+1929$, which differs from my result by $2.5 \sigma$. Reid et al. (2002), on the other hand, found $v_{\text {rad }}=54.1 \pm 0.80 \mathrm{~km} \mathrm{~s}^{-1}$ for $2 \mathrm{M} 0746+2000$, in excellent agreement with my result. Recall that my results could have an additional systematic error of up to $1 \mathrm{~km} \mathrm{~s}^{-1}$ (Sect. 2.2).

I should note that Basri et al. (2000) have observed G1406 to have a significantly different radial velocity of around $40 \mathrm{~km} \mathrm{~s}^{-1}$ on one epoch (June 1997), although they found a constant $18-19 \mathrm{~km} \mathrm{~s}^{-1}$ at several other epochs. They argue that this anomaly is real, perhaps due to stellar pulsations or a companion in a highly elliptical orbit. I therefore checked the radial velocity of G1406 against two other standards observed, G1402 and G1876. These give $18.8 \pm 0.1 \mathrm{~km} \mathrm{~s}^{-1}$ and $18.2 \pm$ $0.5 \mathrm{~km} \mathrm{~s}^{-1}$ respectively (adopting $v_{\text {rad }}=-1.04 \pm 0.03 \mathrm{~km} \mathrm{~s}^{-1}$ for G1402 from Nidever et al. 2002, and $v_{\text {rad }}=-2.0 \pm 0.5 \mathrm{~km} \mathrm{~s}^{-1}$ for Gl876 from Delfosse et al. 1998). This is slightly lower than the adopted value for G1406. Although the difference is significant compared to the random errors, it is at a level where systematic errors could dominate. Moreover, it has no statistically significant impact on the derived radial velocity for the secondary template $2 \mathrm{M} 1439+1929$ : cross correlation against the G1402 and G1876 spectra yields $-26.6 \pm 1.3 \mathrm{~km} \mathrm{~s}^{-1}$ and $-27.3 \pm 1.2 \mathrm{~km} \mathrm{~s}^{-1}$ respectively, compared to the adopted value of $-26.3 \pm 0.50 \mathrm{~km} \mathrm{~s}^{-1}$.

\section{Rotational broadening via cross correlation}

UCD rotation velocities are determined via a cross correlation of the UCD dwarf spectra (the "targets") against spectra of stars 
with essentially zero rotation velocity (the "templates"). Under the assumption that the line broadening of the UCD is dominated by the rotational broadening (and that the line broadening of the template is comparatively small), the width of the cross correlation function is a measure of the $v \sin i$ of the UCD, which may then be calibrated as discussed below. This follows the method used by Tinney \& Reid (1998), Mohanty \& Basri (2003) and White \& Basri (2003). The cross correlation is done on each order separately and the results combined to obtain the final $v \sin i$ measure.

The two primary templates are G1402, an M4V with $v \sin i<2.3 \mathrm{~km} \mathrm{~s}^{-1}$, and G1406, an M5.5V with $v \sin i<$ $2.9 \mathrm{~km} \mathrm{~s}^{-1}$ (Delfosse et al. 1998). The procedure implicitly assumes that the UCDs have similar spectra to the templates, ideally differing only in $v \sin i$. This is of course not the case, and the differences between mid M dwarfs and early/mid L dwarfs could bias the results. But in the absence of appropriate $\mathrm{L}$ dwarfs with independently determined rotation velocities this is the best we can presently do with the cross correlation method. (An alternative method is to examine individual lines according to a physical model of line formation and achieve a best fit against a grid of model spectra, as done, for example, by Schweitzer et al. 2001, although this was not very sensitive to $v \sin i$ ). In some cases, poor fits to the cross correlation peak occur, and such fits are rejected. The impact of spectral type mismatch is assessed via a "bootstrap" method, namely by using the slowest rotating $\mathrm{L}$ dwarf as a secondary template (see Sect. 5).

The cross correlation is done again with FXCOR, but independently of the radial velocity determination. The main peak of the cross correlation function is fit with a Gaussian and its FWHM measured. If a good fit cannot be obtained, or if the peak is ambiguous, then the order is not used.

The main uncertainty in performing this fit is determining the "background" for fitting the Gaussian (i.e. the effective zero level in the cross correlation function). For this reason, three Gaussians were fit corresponding to the best fit, the minimum $F W H M$ fit and the maximum $F W H M$ fit. The fits were performed interactively, with the maximum and minimum representing conservative limits. The range between these comprises a generous assessment of errors in the fitting procedure. Although the peaks sometimes deviated from a Gaussian shape (especially at larger rotation velocities), a Gaussian was nonetheless felt to be the best overall parametrized form among those tested.

These $F W H M$ values are converted to $v \sin i$ values through the following calibration process. The template spectral orders are artificially spun up by convolving their spectra with the rotation profile given by Gray (1992, Eq. (17.12)) with $v \sin i$ spanning 10 to $100 \mathrm{~km} \mathrm{~s}^{-1}$ in steps of $5 \mathrm{~km} \mathrm{~s}^{-1}$. A limb darkening parameter of $\epsilon=0.6$ is assumed in this profile. The spun up spectra are cross correlated with the non-rotating template and the $F W H M$ of the peak is measured in the same way as described above. (Here the difference between the FWHM of the minimum and maximum Gaussian fits is much smaller than in the UCD case, so is neglected.) A linear least squares fit is then obtained between the $F W H M$ and $v \sin i$, separately for each order. It was found that the Gaussian was a poor fit to this cross correlation function above $v \sin i=70 \mathrm{~km} \mathrm{~s}^{-1}$, so the fit is only made in the range $10-70 \mathrm{~km} \mathrm{~s}^{-1}$. For a few orders a good calibration could not be obtained so these were not used.

When this calibration is applied to determine $v \sin i$ for each order and these combined (next section), we must pay attention to the minimum value of $v \sin i$ to which this procedure is sensitive. Following Tonry \& Davis (1979), the measured width of the cross correlation function is made up of a number of components

$\sigma_{\text {meas }}^{2}=\sigma_{\text {rot }}^{2}+\sigma_{\text {nat }}^{2}+2 \sigma_{\text {inst }}^{2}$

where $\sigma_{\text {rot }}$ is the rotational width, $\sigma_{\text {nat }}$ is the natural, or intrinsic, line width in the UCD, and $\sigma_{\text {inst }}$ is the instrumental broadening (two contributions as two spectra form the cross correlation function). I assume that the rotational and intrinsic contributions from the template are negligible. Because the terms are additive, then with "perfect" data, any non-zero rotational broadening could be detected even with non-zero $\sigma_{\text {inst }}$. In reality, however, small broadenings cannot be detected due to noise and template/target mismatch. I make the somewhat ad hoc assumption that we can only detect $\sigma_{\text {rot }}$ if it exceeds the other broadening contributions. If we further assume that the UCD natural broadening is negligible (reasonable if resonance lines and gravity sensitive lines are avoided), then the minimum detectable value of $\sigma_{\text {rot }}$ is equal to $\sqrt{2} \sigma_{\text {inst }}$. The $F W H M$ of the instrumental broadening is set by the slit width of $7.8 \mathrm{~km} \mathrm{~s}^{-1}$ (equal to 1"; when the seeing was below this - as was often the case - the instrumental broadening is reduced). Note that the full width of a rotational profile corresponds to twice the rotational velocity (one half of the line is created by the blueshifted approaching limb of the star, the other half by the redshifted receding limb). Thus the minimum detectable $v \sin i$ is therefore $\sqrt{2} \times 7.8 / 2=5.5 \mathrm{~km} \mathrm{~s}^{-1}$. Any derived values of $v \sin i$ below this limit are dropped from the calculations in the next section. If this limit has been overestimated, then we would potentially drop too many measures, overestimating the combined $v \sin i$. (It turns out that I only drop one value for being below this limit, so this is not significant.) If this limit has been underestimated, then additional values may need to be dropped, potentially raising $v \sin i$.

\section{Projected rotational velocities}

The calibrations described in the previous section are applied to each order to determine a $v \sin i$ for each of the three fits. The different orders are then combined to give the mean, minimum and maximum values as listed in Table 2 . The mean value is a weighted mean of the "best fit" $v \sin i$ values described in the previous section, using a weight of $1,2,3$ or 4 depending on the quality of the fit to its cross correlation peak. Obvious outliers are clipped when forming this mean (7\% of all orders/spectra are clipped in this way). The minimum and maximum values listed in Table 2 are likewise a weighted mean of the minimum and maximum derived $v \sin i$ values.

As discussed in the previous section, the cross correlation method implicitly assumes that the template and target spectra differ only in the rotation velocity, yet the templates are mid $\mathrm{M}$ dwarfs whereas the targets range in spectral type 
from M9 to L5 with one L7.5. This potentially biases the $v \sin i$ determinations. To partially overcome this, the entire cross correlation and calibration procedure is repeated using the slowest rotating $\mathrm{L}$ dwarf as a template. The relevant star is $2 \mathrm{M} 1439+1929$, which was also used as a template by Basri et al. (2000) for the same reason and purpose. Fortunately, this L1.0 dwarf is bright enough to use as a template and is a reasonably early $\mathrm{L}$ type such that its cross correlation against the mid $\mathrm{M}$ dwarfs was probably not too erroneous. Of course, with $\overline{v \sin i}=11.2 \mathrm{~km} \mathrm{~s}^{-1}$, this L dwarf is rotating faster than the $\mathrm{M}$ dwarf templates. Using a rotating template will generally underestimate $v \sin i$, because when we spin up the templates for the calibration, a given $v \sin i$ corresponds to a higher $F W H M$ than would be the case with a non-rotating template. The derived $v \sin i$ values using $2 \mathrm{M} 1439+1929$ as the template are shown in Table 2. Other than in one case (and that is a single measure of lowest quality), there is actually no significant tendency for $v \sin i$ to be lower (or higher) than the values derived with the $\mathrm{M}$ dwarf templates. Indeed, the agreement between the values is good, deviating by less than $2 \mathrm{~km} \mathrm{~s}^{-1}$ in 10 out of 14 cases. In two cases (DE1431-1953 and DE1159+0057) the disagreement is more than $10 \mathrm{~km} \mathrm{~s}^{-1}$, but in both of these cases one of the measurements is based on only a single (poor quality) measurement. In the remaining two cases (2M0913+1841 and SD1203+0015) the discrepancy is around $5 \mathrm{~km} \mathrm{~s}^{-1}$ but the minimum to maximum ranges just about overlap (and both stars are among the faintest observed). Thus although the $v \sin i$ calibration using 2M1439+1929 has used the $\mathrm{M}$ dwarf calibration to identify this template, the spectra and fitting procedures are independent. The fact that both templates give consistent $v \sin i$ ranges indicates that the impact of template mismatch it not major, at least not when using multiple orders across a wide wavelength range ${ }^{3}$. Figure 1 further shows that there is relatively little mismatch between even an M5.5V and L3.0V. Only three of the UCDs are later than this. For the latest type (L7.5V), no acceptable cross correlation function could be obtained against the $\mathrm{M} 5.5 \mathrm{~V}$, perhaps indicative of commencing mismatch problems. In the rest of this article I adopt the $2 \mathrm{M} 1439+1929$ cross correlation values, except of course for 2M1439+1929 itself and except for DE1159+0057 because it has only a single poor quality measurement against this template.

The most striking result from Table 2 is that $\mathrm{L}$ dwarfs are relatively fast rotators, considerably faster than is typically found for $\mathrm{M}$ dwarfs. They all have $\overline{v \sin i}$ in the range $11-37 \mathrm{~km} \mathrm{~s}^{-1}$. Moreover, the lowest value of $\overline{v \sin i}$ is $11.2 \mathrm{~km} \mathrm{~s}^{-1}$, and the lowest minimum value is $9.4 \mathrm{~km} \mathrm{~s}^{-1}$, well above the minimum detection limit of $5.5 \mathrm{~km} \mathrm{~s}^{-1}$ (Sect. 4). This agrees with the results of Mohanty \& Basri (2003), who found no L dwarf in their sample of 13 to have $v \sin i$ below $10 \mathrm{~km} \mathrm{~s}^{-1}$. Two specific targets, $2 \mathrm{M} 0746+2000$ and 2M1439+1929, have

\footnotetext{
${ }^{3}$ This consistency could be misleading if use of the L template gives a positive bias to the derived $v \sin i$ (compared to the $\mathrm{M}$ template) which is then offset by a negative bias from having used a rotating $\mathrm{L}$ template. However, it is not obvious that the later-type template should give a positive bias and, moreover, the two biases would have to contrive to have the same magnitude.
}

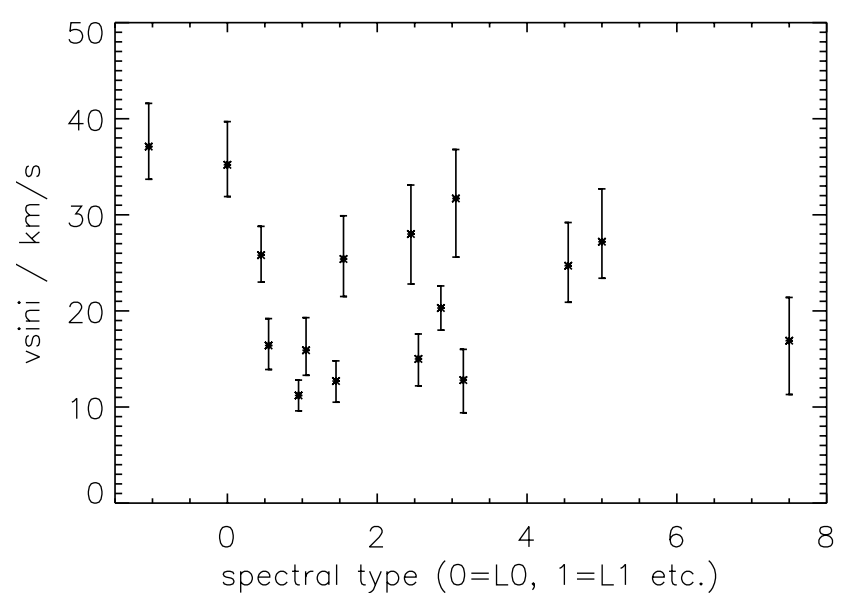

Fig. 2. Projected rotational velocities for ultra cool dwarfs observed in this survey. The values are for the cross correlation against the L dwarf template, 2M1439+1929 (Cols. 8-10 of Table 2) except for 2M1439+1929 itself and DE1159+0057, for which results against the $\mathrm{M}$ dwarf templates are shown. The cross symbol shows the expected value and the "error bars" are the maximum and minimum values. Objects with equal spectral types are slightly offset on the spectral type axis for clarity.

$v \sin i$ determined by others: The former has $24 \pm 5 \mathrm{~km} \mathrm{~s}^{-1}$ according to Reid et al. (2002) and $20 \pm 10 \mathrm{~km} \mathrm{~s}^{-1}$ according to Schweitzer et al. The latter is derived to have $10 \pm 2.5 \mathrm{~km} \mathrm{~s}^{-1}$ by Basri et al. (2000) and $10 \pm 2 \mathrm{~km} \mathrm{~s}^{-1}$ by Mohanty \& Basri (2003). These agree well with the values and ranges derived in the present work.

The projected rotational velocities show no trend with spectral type (Fig. 2), as is also seen in the results from Mohanty \& Basri (2003) over this spectral type range. There is similarly no correlation with $I$ magnitude.

\section{Limits on rotation periods}

The projected rotation velocity, $v \sin i$, is a lower limit to the equatorial rotation velocity, $v$. Assuming that a star rotates as a rigid sphere, $v=2 \pi R / T$, where $R$ is the equatorial radius of the star and $T$ is its rotation period. Given $R$, we can therefore derive the maximum rotation period, $T_{\max }$. More usefully, we can also derive the expected period and "likely" range of periods consistent with $v \sin i$. This may then be compared with published periods obtained from photometric monitoring.

Rigid body rotation is conventionally supported by the theoretical argument that these cool stars are probably fully convective (e.g. Chabrier \& Baraffe 1997) although counter arguments exist (Mullan \& MacDonald 2001). Evolutionary models show that a few hundred million years after formation, UCDs with masses between $0.04 M_{\odot}$ and $0.09 M_{\odot}$ all have radii around $0.1 R_{\odot}$ (Chabrier et al. 1997). Specifically, between $0.5 \mathrm{Gyr}$ and $1.0 \mathrm{Gyr}$ the radii are in the range $0.10-0.12 R_{\odot}$, shrinking to $0.085-0.115 R_{\odot}$ at $5 \mathrm{Gyr}$. As the observed field UCDs reported here are probably at least $1 \mathrm{Gyr}$ old (Gizis et al. 2000), I adopt a radius of $0.1 R_{\odot}$ for all targets when deriving period ranges. The effect of deviations from this are discussed below, as are limits on $R$ which can be derived 
Table 3. Expected, $\langle T\rangle$, maximum, $T_{\max }$, and "minimum", $T_{\min 90}$, rotation periods for the ultra cool dwarfs taking into account both the statistical distribution of $i$ and the range of $v \sin i$ given in Table 2. $\langle T\rangle$ is calculated using the mean $v \sin i$ and the expected value of $\sin i$ (= $\pi / 4$ ). $T_{\max }$ is derived from the minimum $v \sin i$ and maximum $\sin i(=1) . T_{\min 90}$ is calculated from the maximum $v \sin i$ and from that value of $i\left(=26^{\circ}\right)$ for which there is a $90 \%$ chance that the inclination lies above this: i.e., there is only a $10 \%$ chance that the period is shorter than $T_{\text {min } 90}$ when adopting the maximum $v \sin i$ (and even less chance when adopting a more likely $v \sin i$ ).

\begin{tabular}{lrrr|rrr}
\hline \hline Name & \multicolumn{6}{c}{ Rotation period / h } \\
\cline { 2 - 7 } & \multicolumn{3}{c}{ M dwarf template } & \multicolumn{2}{c}{ 2M1439+1929 template } \\
& \multicolumn{1}{c}{$T_{\min 90}$} & $T_{\max }$ & $\langle T\rangle$ & $T_{\min 90}$ & $T_{\max }$ \\
\hline DE1431-1953 & 2.02 & 1.01 & 2.75 & 2.58 & 1.28 & 3.60 \\
DE1159+0057 & 2.72 & 1.34 & 3.81 & 1.28 & 0.64 & 1.76 \\
2M0746+2000 & 3.50 & 1.73 & 4.74 & 3.71 & 1.84 & 5.28 \\
2M1412+1633 & 5.53 & 2.76 & 8.26 & 5.83 & 2.76 & 8.74 \\
2M1439+1929 & 8.54 & 4.14 & 12.65 & - & - & - \\
DE1441-0945 & 5.50 & 2.57 & 8.21 & 6.02 & 2.75 & 9.13 \\
2M1145+2317 & 7.65 & 3.68 & 11.57 & 7.53 & 3.58 & 11.57 \\
2M1334+1940 & 3.80 & 1.89 & 5.50 & 3.77 & 1.77 & 5.65 \\
2M1029+1626 & 3.38 & 1.63 & 5.15 & 3.42 & 1.60 & 5.33 \\
DE1047-1815 & 5.87 & 2.90 & 8.10 & 6.38 & 3.01 & 9.96 \\
2M0913+1841 & 6.38 & 3.01 & 10.21 & 4.71 & 2.35 & 6.75 \\
SD1203+0015 & 3.47 & 1.64 & 5.04 & 3.02 & 1.44 & 4.74 \\
2M1615+3559 & 7.90 & 3.84 & 11.35 & 7.47 & 3.32 & 12.92 \\
2M2224-0158 & 3.77 & 1.90 & 5.47 & 3.87 & 1.82 & 5.81 \\
2M1507-1627 & 3.53 & 1.68 & 5.37 & 3.52 & 1.62 & 5.19 \\
2M0825+2115 & - & - & - & 5.66 & 2.48 & 10.75 \\
\hline
\end{tabular}

from the data. In useful units, the relation between $v \sin i, T$ and $R$ is

$T / \mathrm{h}=121.47 \frac{R}{0.1 R_{\odot}} \frac{1}{v / \mathrm{km} \mathrm{s}^{-1}}$.

Assuming that the spin axes of L dwarfs are randomly oriented in three-dimensional space, it can be seen that the probability, $\mathrm{P}(i) \mathrm{d} i$, of observing a spin axis between $i$ and $i+\mathrm{d} i$ is $\sin i \mathrm{~d} i$. From this we may simply derive that $\langle\sin i\rangle=\pi / 4=0.785$, and hence that $\langle v\rangle=1.27\langle v \sin i\rangle$ from which we calculate $\langle T\rangle$ from the above equation. The largest possible value of $T, T_{\max }$, occurs when $\sin i=1.0$, i.e. when the star is viewed edge on. The minimum value of $T$ is arbitrarily small (as in the limit as $\sin i$ tends toward zero $v$ tends toward infinity). A more useful statement concerns the probability, $f$, that $v$ is not above some value, $v_{f}$. By fixing $f$ at 0.9 we may then say that $v$ is less than $v_{f}$ with $90 \%$ confidence. Generally,

$v_{f}=\left(1-f^{2}\right)^{-1 / 2} v \sin i$

so for $f=0.9, v_{f}=2.29 v \sin i$. The corresponding $90 \%$ confidence lower limit on the period, $T_{\min 90}$, can then be determined from Eq. (2).

These period limits are calculated from the $v \sin i$ values in Table 2 to give the period ranges reported in Table 3 and Fig. 3: the maximum period, $T_{\max }$, is calculated from the minimum value of $v \sin i$, and the minimum period, $T_{\min 90}$, is calculated using the maximum value of $v \sin i$. The expected period, $\langle T\rangle$, is calculated from the mean value of $v \sin i$. Formally, the range $T_{\min 90}: T_{\max }$ is the $90 \%$ confidence interval for the period, and is one-sided because $T_{\max }$ is an absolute maximum (for fixed radius). However, $T_{\min 90}$ is a rather conservative lower limit to the period because the statistical projection argument has been applied to the upper limit of $v \sin i$.

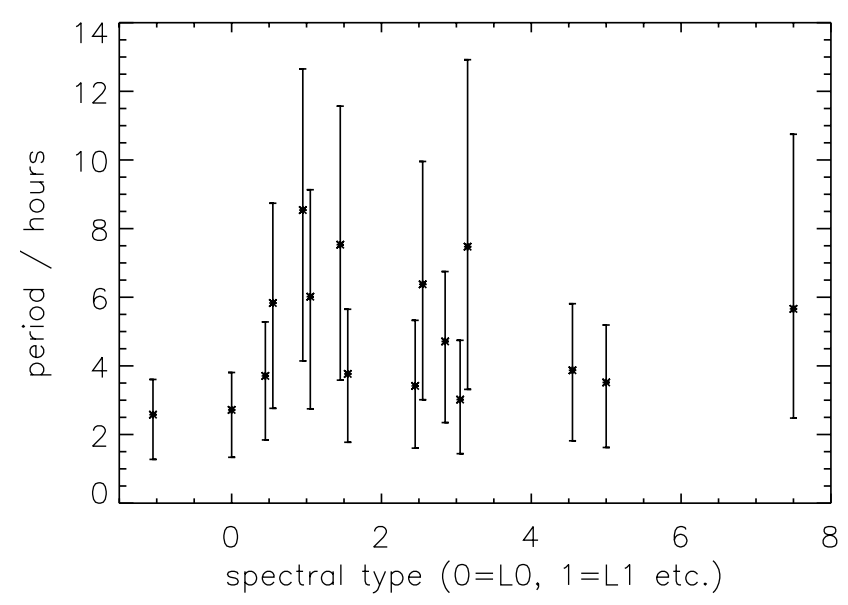

Fig. 3. Derived period limits for the ultra cool dwarfs from the data given in Table 3 for the 2M1439+1929 template (except for 2M1439+1929 and DE1159+0057, which are for the M dwarf templates). The star symbol is the expected period; the "error bars" show the maximum period and $90 \%$ confidence "minimum" period.

\section{Assessment of published variability and period data: Individual objects}

Of the $16 \mathrm{UCDs}$ with $v \sin i$ measured in this work, nine have variability or period data published by one or more authors: Bailer-Jones \& Mundt (2001, hereafter BJM); Bailer-Jones \& Lamm (2003); Clarke et al. (2002a,b, hereafter COT); Gelino et al. (2002, hereafter G02); Gelino (2002). A further five UCDs have variability/period data for which $v \sin i$ values have been published by others. The data for these 14 UCDs are now compared. All variability data is in an $I$ band unless stated otherwise. 
2M0746+2000. This was identified by Reid et al. (2001) as a near equal mass binary with a separation of $0.22^{\prime \prime}$ (2.7 AU) through HST imaging, with a magnitude difference of $\Delta I=0.6$. There is no evidence for binarity from my spectral data which is presumably dominated by the brighter component. COT identify significant variability in their light curve data with an amplitude of 0.007 mag and derive a period of about $3 \mathrm{~h}$, although they underline that this interpretation may be complicated by the binarity. It is also based on just a single dimming in the data (the source was only monitored for $6.5 \mathrm{~h}$ ). Nonetheless, this period is consistent with the period limits in Table 3. G02 report a period of $31 \mathrm{~h}$ for this source with a confidence of almost $5 \sigma$. This is inconsistent with the much smaller maximum period of $5.7 \mathrm{~h}$ in Table 3. This maximum is based on a cross correlation peak clearly widened above the the minimum $v \sin i$ threshold and there is good consistency between the individual orders and the two templates. The $31 \mathrm{~h}$ period of G02 can therefore be ruled out as the rotation period. The rotation period and $v \sin i$ could only be made consistent if the star had a radius of at least $0.6 R_{\odot}$. This would require the star to be either much more massive than an $\mathrm{L}$ dwarf or very young, perhaps only a few million years from its birth line (Chabrier \& Baraffe 1997; see also Joergens et al. 2003). However, in a detailed study based on kinematics, spectroscopy and photometry, Reid et al. (2000) conclude that $2 \mathrm{M} 0746+2000$ has a mass between $0.07 M_{\odot}$ and $0.09 M_{\odot}$ and an age of at least 1 Gyr. A probable explanation for the 31 hour period from G02 is that it is not a real period (a $5 \sigma$ detection is not large for a periodogram). Alternatively, it could be an alias of a shorter period. A third possibility is that this period is not related to the rotation at all, and may not even be a stable period. It could instead be an artifact of unstable light curve modulation due to non-static surface features, as was suggested for some UCDs in BJM. Gelino (2002) also reports $J, H, K$ monitoring observations of this star. Although there is formally no variability across the full light curves, there is strong evidence for a rise of $0.02 \mathrm{mag}$ in $J$ and $K$ lasting $1.5 \mathrm{~h}$ (over a total time span of $5.5 \mathrm{~h}$ : see Fig. 4.5 in that work $)^{4}$. Interestingly, this rise is similar in duration to the dip seen in the same object by COT.

2M1412+1633. G02 report a non-detection of variability on scales above $0.025 \mathrm{mag}$ ( 1.4 times the reported rms) on time scales of up to 85 days (although most data were obtained over 7 days according to Fig. 3.7 of Gelino 2002). Likely periods from Table 3 could in principle have been detected, although the time sampling of G02 is very sparse so sensitivity to the likely periods is low. If there were sufficient temporal sensitivity, then any net rotational modulation must have been smaller than $0.025 \mathrm{mag}$.

2M1439+1929. This was not detected as variable by any of three independent monitoring programs: BJM derive an upper limit of $0.01 \mathrm{mag}$ over timescales of $1 \mathrm{~h}$ to $100 \mathrm{~h}$; a slightly higher upper limit over longer timescales comes from G02.

\footnotetext{
${ }^{4}$ Gelino states that this is not significant, but this conclusion appears to be based on comparing the amplitude with the photometric error in a single measurement. Yet the $1.5 \mathrm{~h}$ rise is observed in about 30 points systematically displaced from the mean, and is clearly a significant deviation.
}

Bailer-Jones \& Lamm (2003) restrict the $J$ and $K$ band variability to be less than $0.04 \mathrm{mag}$ on time scales between $20 \mathrm{~min}$ and 13 days. All of these programs had sampling dense enough to detect likely rotation periods from Table 3 . I conclude that the amplitude of rotational modulation is less than $0.01 \mathrm{mag}$ in $I$ and 0.04 mag in $J$ and $K$.

2M1145+2317. While BJM reported a period of $11.2 \pm$ $0.8 \mathrm{~h}$, a second monitoring epoch reported in the same paper confirmed the variability but not the period. This lack of stability led BJM to suggest that the modulating pattern on the star was not stable: if surface features were evolving during the second epoch on a timescale less than the rotation period, this could have "masked" the rotation period. The present $v \sin i$ data suggest a period between $3.6 \mathrm{~h}$ and $11.6 \mathrm{~h}$, consistent with the $11.2 \mathrm{~h}$ period and this masking hypothesis. (It is not possible that the modulating features were simply weaker in the second epoch as then no variability would have been found.) A period of $11.2 \pm 0.8 \mathrm{~h}$ corresponds to an equatorial rotation speed of $10.8 \pm 0.8 \mathrm{~km} \mathrm{~s}^{-1}$ (Eq. (2)). Combining this with the $v \sin i$ range of $10.5-14.8 \mathrm{~km} \mathrm{~s}^{-1}$ implies that the inclination angle, $i$, lies in the range $62^{\circ}-90^{\circ}$, with this lower limit being rather conservative. The expected value is $i=76^{\circ}$. Thus if the rotation period of $11.2 \pm 0.8 \mathrm{~h}$ from BJM is correct, $2 \mathrm{M} 1145+2317$ would appear to be a near edge-on rotator (which makes detection of rotation by monitoring more likely). If the stellar radius were larger, this would decrease the expected and minimum inclination angles (and eventually the maximum too), and vice versa. Alternatively, we can use this period plus the minimum value of $v \sin i\left(10.5 \mathrm{~km} \mathrm{~s}^{-1}\right)$ to place a lower limit on the radius (i.e. corresponding to $i=90^{\circ}$ ) of this L1.5 dwarf. This gives $R_{\min }=0.097 \pm 0.007 R_{\odot}$, the uncertainty arising from the uncertainty in the period. This is consistent with structure models for a large range of ages (see Sect. 6).

2M1334+1940. The variability detection of this object was one of the most significant in BJM; the detected period of $2.68 \pm 0.13 \mathrm{~h}$ was relatively significant $(12 \sigma)$. This is consistent with the present period deductions and corresponds to an equatorial rotation speed of $45.3 \pm 2.2 \mathrm{~km} \mathrm{~s}^{-1}$. Combining this with the $v \sin i$ range from Table 2 of $21.5-29.9 \mathrm{~km} \mathrm{~s}^{-1} \mathrm{im}-$ plies a range of $i=27^{\circ}-44^{\circ}$, with $i=34^{\circ}$ the expected value. Although this star is quite faint, we can have some confidence in this given the agreement in $v \sin i$ values for the two templates and the reasonable significance of the period detection. As in the previous case, we can instead use the data to derive the minimum radius, which is $R_{\min }=0.047 \pm 0.002 R_{\odot}$. This is a smaller limit than in the previous case, because these data permit smaller inclination angles for 2M1334+1940.

2M1029+1626. G02 report no variability in this L2.5 dwarf, although as this is based on only a few observations per night (Fig. 3.4 of Gelino 2002), this program would probably not have been sensitive to the short periods deduced in Table 3.

2M0913+1841. This was found to be variable by BJM but with no significant period. These observations should have been sensitive to the likely periods reported here (although plausibly the sampling in BJM could have thwarted its detection: see Fig. 1 in that article). Assuming that this variability 
is due to changes in photospheric features, then these features must be changing in pattern and/or brightness at least as fast as the likely rotation periods $(3-10 \mathrm{~h})$ in order to mask a rotationally modulated signature. This is the conclusion of BJM based on the assumption that this L dwarf had a rotation period in the range of detectability. That this is now demonstrated to be likely adds some strength to that conclusion.

SD1203+0015. As with 2M0913+1841, this was found to be a non-periodic variable by BJM. On the other hand, it was intensively monitored by Bailer-Jones \& Lamm (2003) in the $J$ and $K$ bands, who found no variability above the larger limit of 0.04 mag over timescales of 20 min to 7 days. The same comments as for 2M0913+1841 apply.

2M2224-0158. G02 report no variability, but as with $2 \mathrm{M} 1029+1626$, there were only a few observations over each of a few nights, so periods of a few hours could have remained undetected.

There are five other UCDs with published $v \sin i$ values which also have variability data in the literature. These are now briefly discussed to complete this survey of UCD rotation and variability data.

2M1146+2230 (L3V). This is an optical binary with a separation of $0.29^{\prime \prime}(7.6 \mathrm{AU})$ and $\Delta I=0.3 \mathrm{mag}$ (Reid et al. 2001). Mohanty \& Basri (2003) determine $v \sin i=32.5 \pm$ $0.2 \mathrm{~km} \mathrm{~s}^{-1}$, presumably for the brighter component. BJM detected marginally significant variability, with possible rotation periods at $5.1 \pm 0.1 \mathrm{~h}(15 \sigma)$ and $3.0 \pm 0.1 \mathrm{~h}(6 \sigma)$. The former is slightly too long for the measured $v \sin i$, although it would be consistent if the radius were $0.13 R_{\odot}$ (more if the star is not observed edge on). This radius is consistent with the evolutionary models, especially if this L dwarf is less than 1 Gyr in age (Chabrier \& Baraffe 1997). The latter period would also be consistent, but is of too marginal significance to warrant further discussion. Neither G02 nor COT detected variability in this source, although the upper limits of both are consistent with the BJM detection. Given the sampling, either BJM or COT could have detected the likely periods, but the weight of evidence is that the modulation contrast was not large enough (or not stable enough) to permit detection.

LP944-20 (M9V). $\quad v \sin i$ was determined to be $28.3 \pm$ $2 \mathrm{~km} \mathrm{~s}^{-1}$ by Tinney \& Reid (1998) and $39 \pm 2 \mathrm{~km} \mathrm{~s}^{-1}$ by Mohanty \& Basri (2003). Tinney \& Tolley (1999) detected variability with an amplitude of $0.04 \mathrm{mag}$ and timescale of $2 \mathrm{~h}$. (With a p value of only 0.02 this would not have been counted as variable on the criteria of BJM or G02, although the fact that the detection is made in two narrow bands compensates for this.) Although these authors did not associate this with a rotation period, it is consistent with the maximum rotation periods of $4.3 \mathrm{~h}$ and $3.1 \mathrm{~h}$ implied by the above $v \sin i$ values.

DE1228-1547 (L4.5V). Tinney \& Tolley (1999) did not detect variability in this, although their observations were in principle sensitive to the periods commensurable with the $v \sin i$ of $22 \mathrm{~km} \mathrm{~s}^{-1}$ reported by Basri et al. (2000) and Mohanty \& Basri (2003). This implies low contrast surface features.

BRI0021-0214 (M9.5V). Tinney \& Reid (1998) established $v \sin i=42 \pm 8 \mathrm{~km} \mathrm{~s}^{-1}$ for this compared to $34 \pm 2 \mathrm{~km} \mathrm{~s}^{-1}$ from Mohanty \& Basri (2003). Martin et al. (2001) detect several periods in this source from $I$ band monitoring, in particular at $4.8 \mathrm{~h}$ and $20 \mathrm{~h}$. Both are too long for the measured $v \sin i$ range unless the radius is at least $0.13 R_{\odot}$ and $0.5 R_{\odot}$ respectively - more if the star is not observed edge on - something which Martin et al. rule out. This is taken as evidence for the masking hypothesis.

Kelu-1 (L2V). This is a very rapid rotator, as reported both by Basri et al. $\left(2000 ; v \sin i=60 \pm 5 \mathrm{~km} \mathrm{~s}^{-1}\right)$ and Mohanty \& Basri (2003; $v$ sin $i=60 \pm 2 \mathrm{~km} \mathrm{~s}^{-1}$ ). Clarke et al. (2002a) detected Kelu-1 to be periodically variable with a period of $1.80 \pm 0.05 \mathrm{~h}$ and peak-to-peak magnitude of $0.011 \mathrm{mag}$. This is consistent with the $v \sin i$ values, and is discussed at length by Clarke et al.

\section{Conclusions}

I have presented $v \sin i$ measurements for 16 ultra cool field dwarfs with spectral types between M9 and L7.5. 14 of these are new, doubling the number of L dwarfs with measured $v \sin i$. 14 UCDs have $v \sin i$ between 10 and $30 \mathrm{~km} \mathrm{~s}^{-1}$, the other two between 30 and $40 \mathrm{~km} \mathrm{~s}^{-1}$. This confirms a previously published result that, compared to the $\mathrm{M}$ dwarfs, $\mathrm{L}$ dwarfs are fast rotators, with implications for their angular momentum evolution. In addition to establishing mean $v \sin i$ values I determined a conservative $v \sin i$ range for each UCD which encompasses the asymmetric uncertainties in fitting the cross correlation peak: this full range is typically $7 \mathrm{~km} \mathrm{~s}^{-1}$.

With theoretically predicted radii of $0.1 R_{\odot}$, the expected rotation periods of these UCDs (i.e. using $\langle\sin i\rangle=\pi / 4$ ) is $3-10 \mathrm{~h}$. The measured $v \sin i$ determines the maximum rotation period. Using the statistical distribution of $i$, a one-sided $90 \%$ confidence range on the period of each UCD can be established: this is $5.3-12.1 \mathrm{~h}$ and $1.8-4.0 \mathrm{~h}$ for $v \sin i=10 \mathrm{~km} \mathrm{~s}^{-1}$ and $30 \mathrm{~km} \mathrm{~s}^{-1}$ respectively. A larger radius for a given $v \sin i$ would imply longer periods.

From this analysis, suggested periods for UCDs from published monitoring programs were assessed. The following conclusions were drawn. The period of $11.2 \pm 0.8 \mathrm{~h}$ for 2M1145+2317 detected by Bailer-Jones \& Mundt (2001) is likely to be the rotation period for this L1.5 dwarf, and the inclination angle is constrained to be $i=62^{\circ}-90^{\circ}$, i.e. a near edge-on rotator. Likewise, the period of $2.7 \pm 0.1 \mathrm{~h}$ detected for the L1.5 dwarf 2M1334+1940 by the same authors is confirmed as the likely rotation period, and the inclination is $i=27^{\circ}-44^{\circ}$. The period of $31 \mathrm{~h}$ detected by Gelino et al. (2002) for the brighter component of the binary 2M0746+2000 can be strongly ruled out as a rotation period. In three cases (2M0913+1841, 2M1145+2317 and SD1203+0015), variability but no period was detected by monitoring programs, even though the present work derives likely periods which should have been detectable by the programs. Although all of these objects are faint, this seems to support the "masking hypothesis", the idea that surface features evolving in distribution and/or brightness faster than the rotation period could mask such a period from being detected by monitoring. The challenge to ongoing work remains to characterise this evolution.

Acknowledgements. I would like to thank Andreas Kaufer for assistance during the observations and for advice regarding the reduction 
of UVES data. The constructive comments from the referee are gratefully acknowledged.

\section{References}

Allard, F., Hauschildt, P. H., Alexander, D. R., Tamanai, A., \& Schweitzer, A. 2001, ApJ, 556, 357

Bailer-Jones, C. A. L. 2002, A\&A, 389, 963

Bailer-Jones, C. A. L., \& Lamm, M. 2003, MNRAS, 339, 477

Bailer-Jones, C. A. L., \& Mundt, R. 1999, A\&A, 348, 800

Bailer-Jones, C. A. L., \& Mundt, R. 2001, A\&A, 367, 218 (BJM) Erratum: A\&A, 374, 1071

Basri, G., Mohanty, S., Allard, F., et al. 2000, ApJ, 538, 363

Burgasser, A. J., Liebert, J., Kirkpatrick, J. D., \& Gizis, J. E. 2002, AJ, 123,2744

Burrows, A., \& Sharp, C. M. 1999, ApJ, 512, 843

Chabrier, G., \& Baraffe, I. 1997, A\&A, 327, 1039

Chabrier, G., Baraffe, I., Allard, F., \& Hauschildt, P. 2000, ApJ, 542, 464

Clarke, F. J., Tinney, C. G., \& Covey, K. R. 2002a, MNRAS, 332, 361

Clarke, F. J., Oppenheimer, B. R., \& Tinney, C. G. 2002b, MNRAS, 335, 1158 (COT)

Clarke, F. J., Tinney, C. G., \& Hodgkin, S. T. 2003, MNRAS, 341, 239

Delfosse, X., Forveille, T., Perrier, C., \& Mayor, M. 1998, A\&A, 331, 581

Enoch, M. L., Brown, M. E., \& Burgasser, A. J. 2003, AJ, 126, 1006

Gelino, C. R. 2002, Ph.D. Thesis, New Mexico State University

Gelino, C. R., Marley, M. S., Holtzman, J. A., Ackerman, A. S., \& Lodders, K. 2002, ApJ, 577, 433 (G02)

Gizis, J. E., Monet, D. G., Reid, I. N., et al. 2000, AJ, 120, 1085
Gray, D. F. 1992, The observation and analysis of stellar photospheres, second edition (Cambridge Univ. Press)

Hall, P. B. 2002, ApJ, 564, L89

Joergens, V., Fernandez, M., Carpenter, J. M., \& Neuhäuser, R. 2003, ApJ, 594, 971

Koen, C. 2003, MNRAS, 346, 473

Liebert, J., Kirkpatrick, J. D., Cruz, K. L., et al. 2003, AJ, 125, 343

Martín, E. L., Zapatero Osorio, M. R., \& Lehto, H. J. 2001, ApJ, 557, 822

Mochnacki, S. W., Gladders, M. D., Thomson, J. R., et al. 2002, AJ, 124, 2868

Mohanty, S., \& Basri, G. 2003, ApJ, 583, 451

Mullan, D. J., \& MacDonald, J. 2001, ApJ, 559, 353

Nakajima, T., Tsuji, T., Maihara, T., et al. 2000, PASJ, 52, 87

Nidever, D. L., Marcy, G. W., Butler, R. P, Fischer, D. A., \& Vogt, S. S. 2002, ApJS, 141, 503

Reid, I. N., Kirkpatrick, J. D., Gizis, J. E., et al. 2000, AJ, 119, 369

Reid, I. N., Gizis, J. E., Kirkpatrick, J. D., \& Koerner, D. W. 2001, AJ, 121,489

Reid, I. N., Kirkpatrick, J. D., Liebert, J., et al. 2002, AJ, 124, 519

Schweitzer, A., Gizis, J. E., Hauschildt, P. H., Allard, F., \& Reid, I. N. 2001, ApJ, 555, 368

Terndrup, D. M., Krishnamurthi, A., Pinsonneault, M. H., \& Stauffer, J. R. 1999, ApJ, 118, 1814

Tinney, C. G., \& Reid, I. N., MNRAS, 301, 1031

Tinney, C. G., \& Tolley, A. J. 1999, MNRAS, 304, 119

Tonry, J., \& Davis, M. 1979, AJ, 84, 1511

White, R. J., \& Basri, G. 2003, ApJ, 582, 1109

Zapatero Osorio, M. R., Caballero, J. A., Béjar, V. J. S., \& Rebolo, R. 2003, A\&A, 408, 663 